\title{
Bypassing the limit in volumetric imaging of mesoscale specimens
}

\author{
Chaohao Chen, ${ }^{a}$ Zhen Liu, ${ }^{b}$ and Dayong Jin ${ }^{\mathrm{a}, *}$ \\ anniversity of Technology Sydney, Institute for Biomedical Materials and Devices, Faculty of Science, Ultimo, \\ New South Wales, Australia \\ ${ }^{b}$ Southern University of Science and Technology, Department of Biomedical Engineering, Shenzhen, China
}

The development of light-sheet microscopy (LSM) $)^{1,2}$ has revolutionized high-resolution imaging of biological organisms in three dimensions (3D) with minimal photodamage ${ }^{3}$ and high temporal resolution, ${ }^{4}$ therefore allowing long-term observation of biological processes. ${ }^{5,6}$ The key is to obtain information on subcellular events at both high spatial and temporal resolutions, but the spatial resolution of current LSM methods has been pixel-limited when each frame of the image is collected under a large field of view from an entire large-volume specimen.

For this reason, as reported in Advanced Photonics, Fei et al. ${ }^{7}$ developed an algorithm to bypass the conventional limit, which enables high-throughput volumetric imaging of mesoscale specimens at cellular resolution. The as-developed method, called subvoxel light-sheet microscopy (SLSM), can circumvent the trade-off between mapping a large-scale specimen and observing single cells at high resolution. Without complicated design in modulated illumination, SLSM exhibits a much higher acquisition throughput than other microscopy modalities. An off-axial and continuous scanning mode has been implemented to achieve fast acquisition of a series of large field-of-view images with each subvoxel shift encoded in 3D. As illustrated in Fig. 1, the subvoxel resolving algorithm is based on the model of image degradation, probability distributions, and subtle spatial correlations of the acquired images.

Generally speaking, it remains challenging to manage the several trade-offs in optical volumetric imaging, as both excitation and emission light can experience some significant loss by the scattering and absorption through deep tissue. Directly increasing the excitation laser

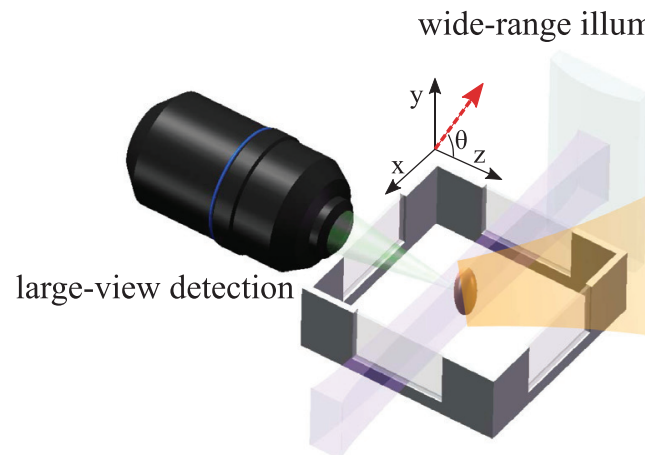

Off-axial sub-voxel scanning

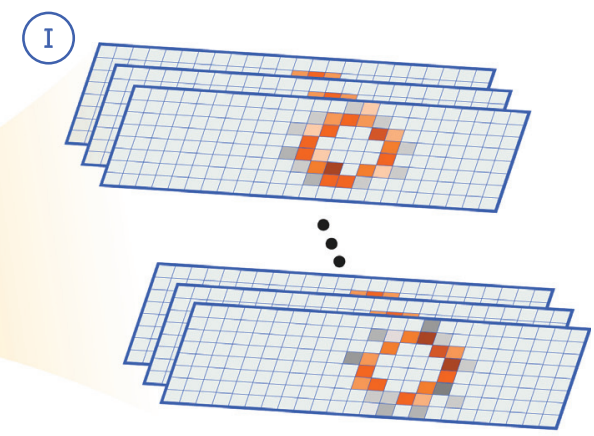

Low resolution images splitting
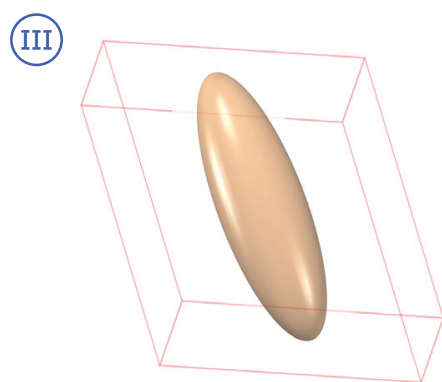

Reshape

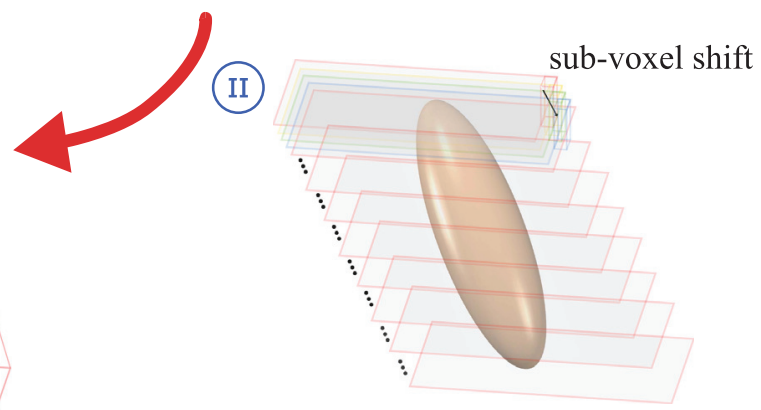

Sub-voxel computational construction

Fig. 1 The principle of subvoxel light-sheet microscopy using wide-range light-sheet illumination and large-view imaging. An off-axial and continuous scanning method is used to achieve fast acquisition of a series of large field-of-view images at low resolution (step I). Through an iterative optimization computational procedure, a voxel super-resolved image can be reconstructed (step II). To compensate the image deformation, voxel realignment is applied (step III).

*Address all correspondence to Dayong Jin, E-mail: dayong.jin@uts.edu.au 
power causes phototoxicity to cells, and increasing the exposure time will decrease the temporal resolution; multiphoton approaches provide high resolution and low phototoxicity but commonly suffer from the low speed and low efficiency of the fluorescent probes.

LSM uses the relatively large area of light sheet shaped from a coherent laser source to illuminate the large-volume sample sideways, layer by layer, which can significantly increase both penetration depth and imaging throughput, and therefore may circumvent some of the above-mentioned key challenges. However, the trade-off when minimizing the thickness of a hyperbolic laser sheet is to sacrifice its confocal range (the area of the field of view). The SLSM approach reported by Fei et al. ${ }^{7}$ takes advantage of the subvoxel resolving algorithm to allow a relatively thick and uniform laser sheet to cover a wide field of view while maintaining the comparable axial resolution that the thin laser sheet illumination can achieve.

Fei and his team reported that SLSM provides a wide field of view as large as $\sim 23 \mathrm{~mm}^{2}$ using a low-magnification $4 \times / 0.13$ detection objective and a low 0.022-NA (numerical aperture) plane illumination configuration, ${ }^{7}$ whereas the achievable resolution is similar to that achieved using $20 \times$ LSM. Circumventing the use of high-NA objectives allows SLSM to obtain fast data acquisition at a rate above two million voxels per second. Fei's team has further developed a GPU-based parallel computation flow to cope with such fast acquisition speed. ${ }^{7}$ Thereby, using a relatively simple small NA configuration, the demonstrated prototype has achieved high-resolution volumetric imaging of mesoscale specimens at high temporal resolution. The reported approach is also compatible with both additional illumination modulations and other fluorescence labels. In SLSM experiments, the multiview fusion approach ${ }^{8}$ can be combined to achieve complete imaging of the highly scattering and thick samples with an isotropic resolution throughout a volume of more than $100 \mathrm{~mm}^{3}$, suggesting its potential in tissue regeneration research and brain imaging.

With the exciting advances presented by SLSM, its resolution can be further improved. When increasing imaging depth in the heterogeneity of multicellular media, the optical resolution is compromised due to the issue of aberration and absorption of light. The deep-tissueintroduced aberrations can be corrected using adaptive optics, ${ }^{6,9}$ and the "self-healing" capability of the Bessel beam ${ }^{10}$ or Airy beam ${ }^{11}$ can minimize the scattering artifacts for high-resolution imaging of cellular structures with varied refractive indices. New developments of multiphoton techniques ${ }^{12}$ and new probes ${ }^{13,14}$ will further advance this field; the remaining challenge is to integrate these advanced techniques in volumetric imaging at high spatiotemporal resolution.

Fei et al.' $\mathrm{s}^{7}$ introduction of the optimization-based iterative algorithm to enable the subvoxel volumetric imaging in $3 \mathrm{D}$ is provocative. Validation becomes very important. As an algorithm solves the illposed inverse problem, the cost function can be biased, or the steepest gradient descent iteration can be stopped at a local extremum since the goal function could be nonconvex. Although Fei's team has presented quite a lot of work to avoid this, it is important that a quantifiable measure, such as peak signal-to-noise ratio or structural similarity index measure, be explored in the future. This work is inspiring to new developments of imaging algorithms for enhancing the performance of conventional LSM. Beyond these, the data-driven approach of deep learning can also be investigated by a well-trained artificial neural network to reconstruct the high-resolution images from a relatively small number of frames, which may bypass the complicated image degradation. The super-resolution images could be obtained in an end-to-end model, even possibly without the image stitching process.

The demonstrated spectacular images reported by Fei et al. ${ }^{7}$ suggest a wide range of potential applications in histology, pathology, and neuroscience. We anticipate this work to be adopted by multidisciplinary research labs to benefit from the latest developments in three-dimensional volumetric imaging modalities.

\section{References}

1. R. M. Power and J. Huisken, "A guide to light-sheet fluorescence microscopy for multiscale imaging," Nat. Methods 14, 360-373 (2017).

2. S. Wolf et al., "Whole-brain functional imaging with two-photon light-sheet microscopy," Nat. Methods 12, 379-380 (2015).

3. W. Zong et al., "Large-field high-resolution two-photon digital scanned light-sheet microscopy," Cell Res. 25, 254-257 (2015).

4. M. B. Ahrens et al., "Whole-brain functional imaging at cellular resolution using light-sheet microscopy," Nat. Methods 10, 413420 (2013)

5. L. A. Royer et al., "Adaptive light-sheet microscopy for long-term, high-resolution imaging in living organisms," Nat. Biotechnol. 34, 1267-1278 (2016)

6. T. L. Liu et al., "Observing the cell in its native state: imaging subcellular dynamics in multicellular organisms," Science 360(6386), eaaq1392 (2018).

7. P. Fei et al., "Subvoxel light-sheet microscopy for high-resolution high-throughput volumetric imaging of large biomedical specimens," Adv. Photonics 1(1), 016002 (2019).

8. J. Swoger et al., "Multi-view image fusion improves resolution in three-dimensional microscopy," Opt. Express 15(13), 8029-8042 (2007).

9. M. J. Mlodzianoski et al., "Active PSF shaping and adaptive optics enable volumetric localization microscopy through brain sections," Nat. Methods 15, 583-586 (2018).

10. F. O. Fahrbach et al., "Light-sheet microscopy in thick media using scanned Bessel beams and two-photon fluorescence excitation," Opt. Express 21(11), 13824-13839 (2013).

11. T. Vettenburg et al., "Light-sheet microscopy using an Airy beam," Nat. Methods 11, 541-544 (2014).

12. A. Song et al., "Volumetric two-photon imaging of neurons using stereoscopy (vTwINS)," Nat. Methods 14, 420-426 (2017).

13. C. Chen et al., "Multi-photon near-infrared emission saturation nanoscopy using upconversion nanoparticles," Nat. Commun. 9, 3290 (2018).

14. M. Kamper et al., "Near-infrared STED nanoscopy with an engineered bacterial phytochrome," Nat. Commun. 9, 4762 (2018).

Chaohao Chen received his MS degree in micro and nano technology systems from the University of South-Eastern Norway in 2016. He is currently a PhD candidate working in the field of nanophotonics at the University of Technology Sydney. His interests include novel superresolution modalities based on upconversion nanoparticles and optofluidic devices.

Zhen Liu received his bachelor's degree in physics from Hunan University and master's degree in optics from Xiamen University in 2010 and 2013, respectively. He has been a research assistant at Southern University of Technology and Science since 2018, after years of engineering experience in the industry.

Dayong Jin is a distinguished professor at the University of Technology Sydney. His research has been in the physical, engineering and interdisciplinary sciences. $\mathrm{He}$ is a technology developer with expertise covering photonics, microscopy, cytometry, materials, and analytical chemistry. He won the Australian Museum Eureka Prize in 2015, the Australian Academy of Science John Booker Medal in 2017, and the Prime Minister's Prize for Physical Scientist of the Year in 2017. 